\title{
Akute Lahmheiten beim Hund - regulationsmedizinisch begleitet
}

\author{
Heidi Kübler
}

In der tierärztlichen Praxis werden Hunde mit Lahmheiten sehr häufig vorgestellt. Neben der konventionellen schulmedizinischen Therapie gibt es eine ganze Reihe von regulationsmedizinischen Therapieansätzen, die als Monotherapie oder begleitend zu weiteren Therapieverfahren eingesetzt werden können und die von Tierhaltern vermehrt nachgefragt werden. Die folgende Übersicht fokussiert sich auf die akuten Probleme am Bewegungsapparat.

\section{Lahmheiten}

Ursache für akute Lahmheiten sind in den meisten Fällen traumatische Ereignisse wie z.B. Stürze, Rempeleien unter Hunden oder Unfälle. Doch auch Überbelastungen durch nicht der Kondition des Hundes angepasste Bewegungsaktivitäten (an den Wochenenden oder in der Urlaubszeit) führen zu Lahmheiten oder Veränderungen im normalen Bewegungsablauf. In vielen Fällen kann der Tierhalter jedoch kein auslösendes Ereignis für eine akute Lahmheit nennen.

Umso wichtiger ist in solchen Fällen eine eingehende Untersuchung zur Lokalisation des Problems, denn es können so verschiedene Strukturen wie Knochen, Muskeln, Sehnen, Bänder oder Nerven geschädigt sein. Insbesondere geht es natürlich darum abzuklären, ob ggf. eine schnelle chirurgische Intervention notwendig ist, damit keine weitreichenden Folgeschäden entstehen.

Bei Welpen und Junghunden in Wachstum und Entwicklung erfordern Lahmheiten besondere Aufmerksamkeit und frühzeitige weiterführende Diagnostik wie z.B. Röntgen, um entwicklungsbedingte Erkrankungen wie IPA, IPC, OCD usw. möglichst frühzeitig zu diagnostizieren und adäquat zu behandeln.

Durch nicht frühzeitig erkannte und korrigierte Entwicklungsstörungen kommt es zu irreversiblen Schäden an Gelenken, die den Hund sein Leben lang begleiten und zu chronischen Schmerzgeschehen führen können.

\section{Schmerz}

Tiere zeigen Lahmheiten oder Schonhaltung, weil sie Schmerzen haben. In der Praxis zeigen sich Lahmheiten häufig nicht so gravierend wie im häuslichen Umfeld, da Hunde dazu neigen, in unbekannter Umgebung oder bei Aufregung vorhandene Schmerzen möglichst nicht zu zeigen.

Bei akut auftretenden Problemen hat Schmerz die physiologische Funktion, für eine Schonung zu sorgen, damit traumatisierte Gewebe während der Regenerationsphase nicht überlastet werden. Insofern ist bei perakut und akut verlaufenden gering- bis mittelgradigen Lahmheiten der Einsatz potenter Analgetika wie z.B. NSAIDs oder Opioiden durchaus kritisch zu sehen.

Auf der anderen Seite aber hat Schmerz die Neigung, zu chronfizieren, wenn keine adäquate Schmerztherapie erfolgt. Aus anhaltenden oder wiederkehrenden Schmerzen können so chronische, nach Beseitigung der auslösenden Ursache weiter bestehende Schmerzen entstehen.

Wird ein akut lahmender Hund mit potenten Analgetika therapiert, muss der Tierhalter unbedingt für Schonung sorgen, denn ein schmerzfreies Tier tobt und rennt wieder wie vor dem schmerzhaften Ereignis, auch wenn Veränderungen in Geweben da sind, die noch ausheilen müssen, damit es keine Spätschäden gibt.

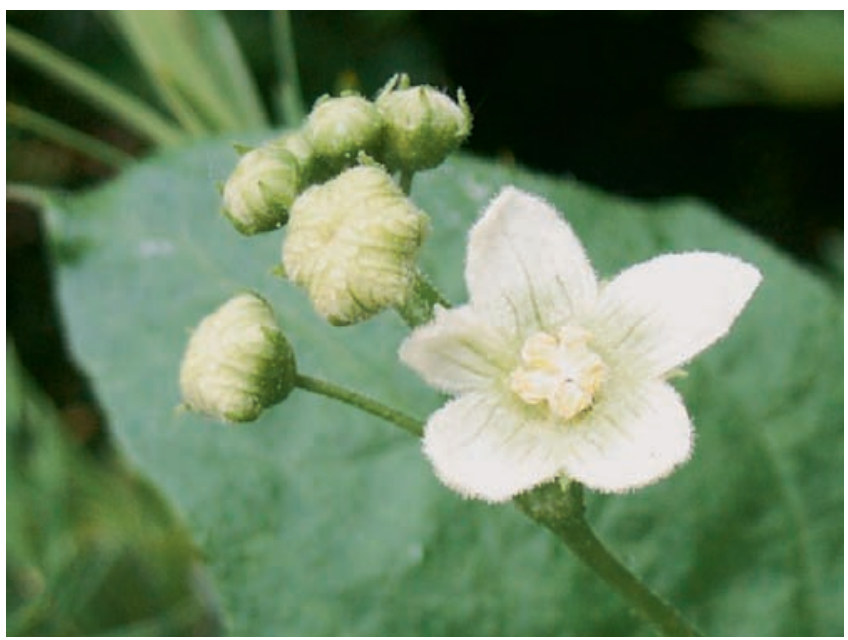

Abb. 1 Bryonia-Blüte

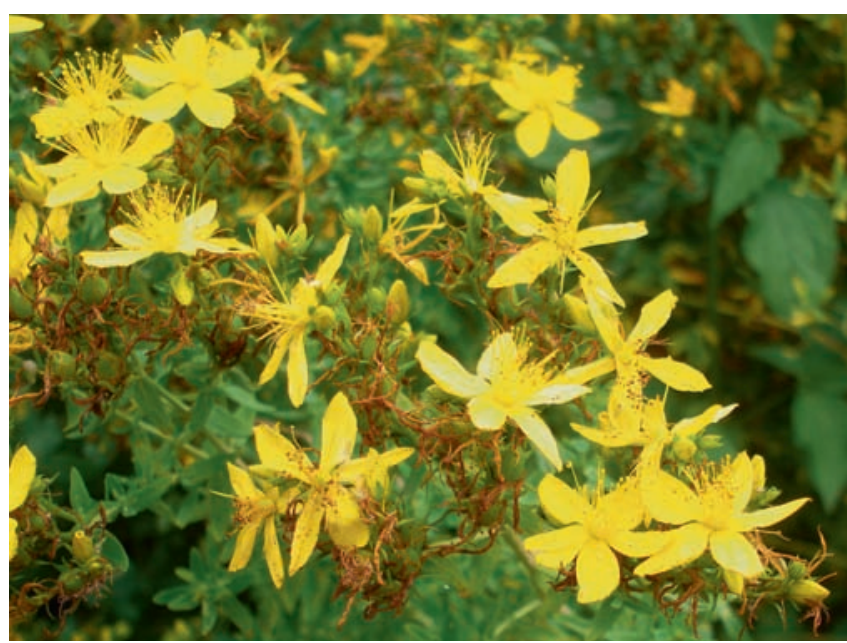

Abb. 2 Johanniskraut-Blüten 


\section{Regulationsmedizin}

Als Bausteine einer multimodalen Schmerztherapie sind Therapieverfahren aus dem Bereich der Regulationsmedizin anzusehen. Sie können bei geringgradigen Schmerzen als alleinige Therapie, bei mittel- bis hochgradigen Schmerzen begleitend oder als Anschlusstherapie an die Therapie mit potenten Analgetika eingesetzt werden.

Sie beseitigen Schmerzen anfangs meist nicht völlig, sondern reduzieren sie auf ein für das Tier erträgliches Maß. Das Tier spürt noch, dass etwas nicht in Ordnung ist und ist eher ruhig zu halten, als wenn es völlig schmerzfrei ist.
Regulationsmedizinische Therapieverfahren haben ein extrem geringes Nebenwirkungspotenzial. Deshalb bieten sich gerade für unkomplizierte akute gering- bis mittelgradige Lahmheiten aufgrund von Verstauchungen, Prellungen, Zerrungen und Quetschungen eine Reihe von regulationsmedizinischen Therapieoptionen an - von homöopathischen Einzelmitteln über Komplexpräparate bis hin zu phytotherapeutischen Zubereitungen. Zur Akutbehandlung in der Praxis eignet sich eine Low-level-Lasertherapie z.B. mit einem Infrarotlaser.

\section{Homo̊opathie}

\section{Homöopathische Einzelmittel}

Bei Lahmheiten werden homöopathische Einzelmittel nach ihrem Arzneimittelbild (Gesamtheit der am Patienten feststellbaren Symptome) und/oder den sog. bewährten Indikationen (klinische Diagnosen, bei denen sich ein bestimmtes Mittel besonders gut bewährt hat) eingesetzt ( Tab. 1 u. Tab. 2).

\section{Dosierung}

Je akuter ein Geschehen aufgetreten ist, desto häufiger erfolgt anfangs die Gabe des ausgewählten Mittels ( Tab. 3).

Tab. 1 Homöopathische Einzelmittel mit Bezug zu Knochen und Gelenken (und in den Fertigpräparaten eingesetzte Potenzen mit entsprechenden Fertigpräparaten).

\begin{tabular}{|c|c|c|c|}
\hline Mittel & $\begin{array}{l}\text { Deutsche } \\
\text { Bezeichnung }\end{array}$ & Charakteristik, Anwendungsgebiete & Enthalten in: \\
\hline Bryonia D4, D6 & Zaunrübe & $\begin{array}{l}\text { Gelenke geschwollen und warm, trockene } \\
\text { Schleimhäute, großer Durst auf kaltes Wasser, } \\
\text { Verschlimmerung durch Bewegung; Arthritis, } \\
\text { Polyarthritis, Distorsion }\end{array}$ & $\begin{array}{l}\text { Bryonia-logoplex }{ }^{\circledR} \text {, } \\
\text { Bryonia-Injeel }^{\circledR}\end{array}$ \\
\hline $\begin{array}{l}\text { Calcium carbonicum Hahne- } \\
\text { manni D12, D30 }\end{array}$ & Austernschalenkalk & $\begin{array}{l}\text { Ruhige Tiere mit kräftigem Knochenbau, Ent- } \\
\text { wicklungsstörungen im Welpenalter; Rachitis, } \\
\text { Arthritis }\end{array}$ & $\begin{array}{l}\text { Calcium carbonicum-Injeel }{ }^{\circledR} \text {, } \\
\text { China-logoplex }^{\circledR}\end{array}$ \\
\hline Calcium fluoratum D6, D12 & $\begin{array}{l}\text { Calciumfluorid, } \\
\text { Flussspat }\end{array}$ & $\begin{array}{l}\text { Rasche ermüdbare, unruhige und hektische } \\
\text { Tiere, Durchtrittigkeit in den Gelenken, } \\
\text { Frakturen heilen schlecht; Arthritis, Osteo- } \\
\text { chondrose, Wachstumsstörungen, Neigung zu } \\
\text { Verstauchungen und Verrenkungen }\end{array}$ & Calcium fluoratum-Injeel ${ }^{\circledR}$ \\
\hline $\begin{array}{l}\text { Calcium phosphoricum D6, } \\
\text { D12 }\end{array}$ & $\begin{array}{l}\text { Calcium-hydrogen- } \\
\text { phosphat }\end{array}$ & $\begin{array}{l}\text { Lebhafte, schlanke und zierliche Tiere, sind } \\
\text { schnell erschöpft, kränklich; Entwicklungs- } \\
\text { und Wachstumsstörungen, „Wachstums- } \\
\text { schmerzen“ }\end{array}$ & $\begin{array}{l}\text { Discus compositum ad us. } \\
\text { vet. }{ }^{\circledR} \text { Osteoheel } S^{\circledR}\end{array}$ \\
\hline $\begin{array}{l}\text { Eupatorium perfoliatum D4, } \\
\text { D6, D8 }\end{array}$ & Wasserhanf & $\begin{array}{l}\text { Starke Knochenschmerzen, großer Durst; } \\
\text { Distorsionen, Frakturen }\end{array}$ & $\begin{array}{l}\text { Belladonna-logoplex }{ }^{\circledR} \\
\text { Echinacea comp. SN }{ }^{\circledR}\end{array}$ \\
\hline Ledum D6, D12 & Sumpfporst & $\begin{array}{l}\text { Heftige, reißende Schmerzen, nach Stich- } \\
\text { oder Bisswunden, Wärme verschlimmert, Käl- } \\
\text { te und kalte Anwendungen bessern; Arthritis, } \\
\text { Bisswunden, Insektenstiche, Zeckenbisse }\end{array}$ & \\
\hline Symphytum D2 & Beinwell & $\begin{array}{l}\text { „Arnica der Knochen“; Bezug zu Knochen und } \\
\text { Periost, bei Neigung zu Exostosenbildung; } \\
\text { Distorsionen, Periostitis, verzögerte Kallusbil- } \\
\text { dung bei Frakturen }\end{array}$ & $\begin{array}{l}\text { Articulatio comp. PLV }{ }^{\circledR} \text {, } \\
\text { Arnica-logoplex }{ }^{\circledR}, \text { Symphy- }^{-} \\
\text {tum-Injeel }^{\circledR} \text { Traumeel }^{\circledR}\end{array}$ \\
\hline
\end{tabular}


Tab. 2 Homöopathische Einzelmittel mit Bezug zu Nerven, Bändern und Muskulatur (und in den Fertigpräparaten eingesetzte Potenzen mit entsprechenden Fertigpräparaten).

\begin{tabular}{|c|c|c|c|}
\hline Mittel & $\begin{array}{l}\text { Deutsche } \\
\text { Bezeichnung }\end{array}$ & Charakteristik, Anwendungsgebiete & Enthalten in: \\
\hline Apis D4, D30 & Honigbiene & $\begin{array}{l}\text { Rote, ödematöse, schmerzhafte Schwellungen, Besse- } \\
\text { rung durch kalte Umschläge, Durstlosigkeit; Arthritis, } \\
\text { Tendinitis/Tendovaginitis }\end{array}$ & Apis-Homaccord ${ }^{\circledR}$ \\
\hline Arnica D3, D4 & Bergwohlverleih & $\begin{array}{l}\text { Extreme Berührungsempfindlichkeit am ganzen Kör- } \\
\text { per, akute Distorsion mit Hämatom und/oder Ödem, } \\
\text { Luxationen, Quetschungen }\end{array}$ & $\begin{array}{l}\text { Arnica e planta tota D5 PLV }{ }^{\circledR} \text {, } \\
\text { Arnica-logoplex }{ }^{\circledast} \text {, Articulatio } \\
\text { comp. PLV }^{\circledR}, \text { Magnesium } \\
\text { comp. PLV }^{\circledast} \text { Traumee }^{\circledR}\end{array}$ \\
\hline Bellis perennis D3, D4 & Gänseblümchen & $\begin{array}{l}\text { Zerschlagenheitsgefühl mit Blutungsneigung, Folge } \\
\text { von Überanstrengung und Überlastung von Muskula- } \\
\text { tur und Bändern, nach stumpfen Traumata }\end{array}$ & Arnica-logoplex ${ }^{\circledast}$ Traumee $^{\circledast}$ \\
\hline Gelsemium D6, D30 & Wilder Jasmin & $\begin{array}{l}\text { Eher ängstliche Tiere, Zittern der Extremitäten bei } \\
\text { Aufregung, schwacher Muskeltonus }\end{array}$ & $\begin{array}{l}\text { Rhus toxicodendron comp. } \\
\text { PLV }{ }^{\otimes}\end{array}$ \\
\hline Hammamelis D4, D6 & Zaubernuss & $\begin{array}{l}\text { Mittel für venöse Sickerblutungen, dunkle Verfärbung } \\
\text { der Haut; Hämatome, Quetschungen }\end{array}$ & Arnica-logoplex ${ }^{\circledR}$, Traumee $^{\circledR}$ \\
\hline Hypericum D4, D6 & Johanniskraut & $\begin{array}{l}\text { „Arnica der Nerven“; Bei Nervenschädigungen, } \\
\text { Neuralgien nach Verletzungen, Operationen, Contusio } \\
\text { cerebri, Neuritis }\end{array}$ & Arnica-logoplex ${ }^{\circledR}$, Traumee $^{\circledR}$ \\
\hline $\begin{array}{l}\text { Rhus toxicodendron D6, } \\
\text { D12, D30 }\end{array}$ & Giftsumach & $\begin{array}{l}\text { Beschwerden bessern sich durch Bewegung, Tiere sind } \\
\text { ruhelos, bei Verstauchungen, Zerrungen, Steifheit des } \\
\text { Bewegungsapparates }\end{array}$ & $\begin{array}{l}\text { Arnica logoplex }{ }^{\circledast}, \text { Rhus toxi- } \\
\text { codendron comp. PLV }{ }^{\circledR} \text {, Rhus } \\
\text { tox-Injeel }{ }^{\circledR}\end{array}$ \\
\hline Ruta graveolens D2, D4 & Weinraute & $\begin{array}{l}\text { Steifheit und Zerschlagenheitsgefühl, bei Prellungen, } \\
\text { Quetschungen, Zerrungen }\end{array}$ & Ruta-Injeel ${ }^{\circledR}$ \\
\hline
\end{tabular}

Es wird empfohlen, bei plötzlich auftretender Lahmheit anfangs für $1-2 \mathrm{~h}$ alle 15-30 min 1 Gabe des ausgewählten homöopathischen Mittels zu verabreichen.

- Bessert sich die Lahmheit nach 4-6 Gaben, wird die Häufigkeit der Gabe reduziert auf alle 2-4h 1 Gabe für 6-8h.

- Bei weiterer Besserung erhält das Tier am Folgetag 2-4 × tägl. 1 Gabe, bis die Lahmheit wieder völlig verschwunden ist.

\section{Homöopathische Komplexmittel}

In Komplexmitteln sind mehrere homöopathische Einzelmittel kombiniert, die eine ähnliche Wirkrichtung oder einen Bezug zu bestimmten Organen oder Geweben haben. Der Einsatz von Komplexmitteln erfolgt überwiegend nach klinischen Diagnosen, weniger nach den homöopathischen Arzneimittelbildern. Deshalb kann die Behandlung akuter Probleme mit Komplexmitteln in jeder Praxis auch ohne langjährige regulationsmedizinische Ausbildung erfolgen.

Beispiele für Komplexmittel zur Verabreichung bei akuten Lahmheiten sind:

- Traumeel $^{\circledR}$ ad us. vet. Ampullen,

Tabletten, Tropfen, Salbe oder Gel

- Arnica-logoplex ${ }^{\circledR}$-Injektionslösung oder Globuli

\section{Dosierung}

Bei akuten Lahmheiten können o.g. Präparate in der Praxis verabreicht werden: - initial als Injektion
- Die Fortsetzung der Behandlung erfolgt oral - anfangs alle 2-4h

1 Gabe, bei Besserung dann noch 2-3 × tägl. 1 Gabe (๑ Tab. 3).

Gerade bei kleinen Hunden hat sich bewährt, die Injektionspräparate vom Tierbesitzer oral verabreichen zu lassen, da die salzig schmeckenden Lösungen von den Tieren gerne akzeptiert werden. Nach 2 Tagen sollte eine Kontrolluntersuchung in der Praxis stattfinden. Hat sich die Lahmheit kaum gebessert, sollten auf jeden Fall weitergehende Untersuchungen eingeleitet werden. Ist der Hund bereits fast lahmheitsfrei, sollte die medikamentelle regulationsmedizinische Therapie noch mindestens 4-6 Tage lang durchgeführt werden, damit das geschädigte Gewebe wieder völlig ausheilt. 
Tab. 3 Dosierung homöopathischer Einzel- und Komplexmittel (Richtdosis).

\begin{tabular}{|c|c|c|c|}
\hline Tiergröße & Globuli & Dilution/Tropfen & Tabletten \\
\hline Welpe $(<1 \mathrm{~kg})$ & 2 & 2 Tropfen & $1 / 4$ \\
\hline Hund (1-5 kg) & 3 & 4 Tropfen & $1 / 2$ \\
\hline Hund $(5-20 \mathrm{~kg})$ & 4 & 6 Tropfen & 1 \\
\hline Hund $(20-40 \mathrm{~kg})$ & 5 & 8 Tropfen & 2 \\
\hline Hund über $40 \mathrm{~kg}$ & 8 & 10 Tropfen & 3 \\
\hline
\end{tabular}

\section{Zubereitungen homöopathischer Präparate}

Es gibt homöopathische Einzelmittel und auch Komplexmittel als Dilutionen (alkoholische Lösungen), Globuli (aus Rohrzucker) und Tabletten (enthalten Milchzucker). Alle Zubereitungsformen sollten dem Patienten möglichst direkt in die Lefzen bzw. auf die Mundschleimhaut verabreicht werden.

\section{Verabreichung von homöopathischen Einzelmitteln und Komplexmitteln}

Da die Homöopathika möglichst intensiven Kontakt mit der Mundschleimhaut haben sollten - denn bereits dort setzt ihre Wirkung ein -, hat es sich bewährt, die Mittel nach Möglichkeit 30 min vor oder nach der Fütterung mit einer Plastik-Einmalspritze, einer Tropfpipette oder einem Plastiklöffel direkt in die Lefzen zu geben.
Sollte eine orale Eingabe für den Besitzer problematisch sein, können die Präparate auch unter eine sehr kleine Menge breiiges bzw. suppiges Futter gegeben werden.

\section{Homöopathika bei Distorsionen, Kontusionen}

Das wichtigste und bekannteste Mittel für akute Traumata aus der Homöopathie ist Arnica. Es wird eingesetzt bei allen Weichteiltraumata, insbesondere wenn es durch Quetschungen zu Einblutungen ins Gewebe mit Hämatombildung kommt und der Hund sehr berührungsempfindlich ist. Auch bei Muskelkater nach Überanstrengung wirkt es schmerzlindernd.

Prä- und postoperativ verabreicht reduziert es die Blutungsneigung. Seine Wirkung entfaltet sich am besten in den ersten 2-4 Tagen nach einer Verletzung. Deshalb sollte es auf keinen Fall eine längere Zeit gegeben werden. Sind danach noch Beschwerden vorhanden, ist meist ein Wechsel des Mittels notwendig.

So kann als Folgemittel Rhus toxicodendron eingesetzt werden, wenn die Hunde direkt nach dem Aufstehen noch stark lah- 


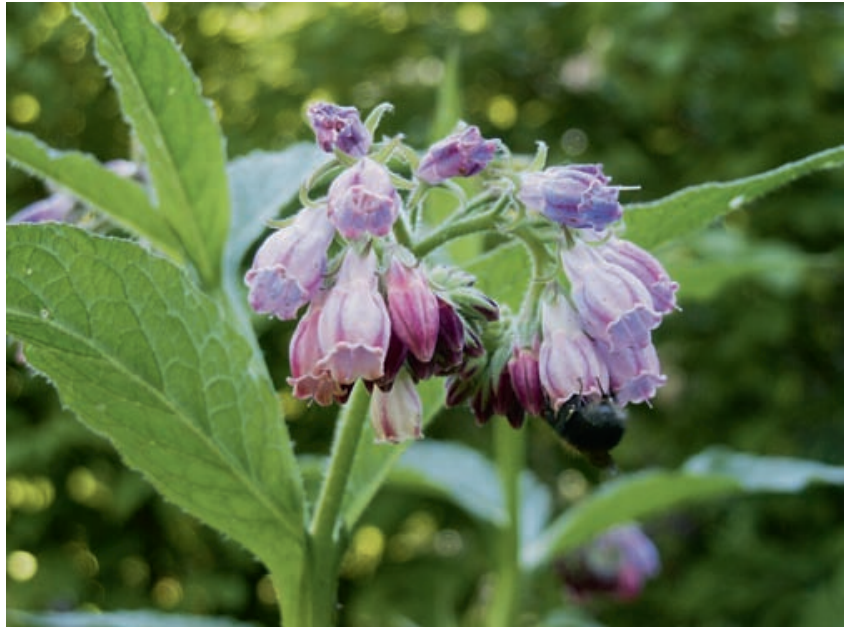

Abb. 3 Beinwell-Blüten

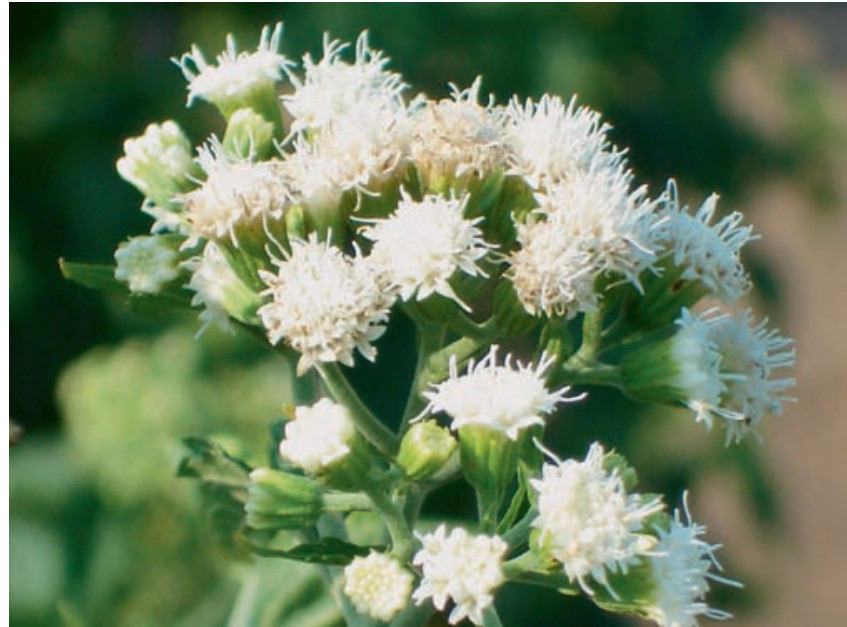

Abb. 4 Wasserhanf-Eupatorium-Blüten men, sich beim Spaziergang nach und nach „einlaufen“ und nach dem Spaziergang fast lahmheitsfrei gehen.

Eine Kombination der 3 homöopathischen Einzelmittel Arnica, Ruta und Rhus toxicodendron kann für akute Schmerzzustände eingesetzt werden, die mit Steifigkeit einhergehen und sich durch fortgesetzte Bewegung wieder bessern.

\section{Dosierung}

2-4 × tägl. 1 Gabe jeden Mittels sollte eine akute Lahmheit innerhalb von 3-4 Tagen zum Verschwinden bringen. Zusätzlich sollte der Hund an der Leine geführt werden und nicht mit anderen toben.

\section{Homöopathische Begleitung orthopädischer Eingriffe \\ Dosierung}

- Am Tag vor der OP: Arnica C30 - einmalige Gabe

- Am Operationstag direkt nach der Narkose: Nux vomica C30 - einmalige Gabe

- Am Operationstag und dem darauffolgenden Tag: Arnica C30 - einmalige Gabe

- Ab 3. Tag bis ca. 14. Tag nach OP: Staphisagria D12 - 2 × tägl. 1 Gabe

\section{Schůßler-Salze}

Die Prophylaxe von wachstumsbedingten Problemen am Bewegungsapparat spielt v.a. bei den großwüchsigen Hunderassen wie Doggen, Schäferhunden, Retrievern usw. eine wichtige Rolle. Zusätzlich zu einer dem Wachstum und der Ent- wicklung angepassten eher restriktiven Fütterung hat sich der Einsatz von Schüßler-Salzen bewährt ( Tab. 4).

Schüßler-Salze sind 12 verschiedene homöopathisch potenzierte Mineralstoffverbindungen, die von dem Arzt Dr. Wilhelm Heinrich Schüßler(1821-1898) um 1870 entwickelt und eingesetzt wurden. Seine Nachfolger ergänzten das Therapiesystem um weitere 12 sog. Ergänzungsmittel.

Es handelt sich um ein Therapieverfahren der Erfahrungsheilkunde, das von
Menschen in den allermeisten Fällen in Selbstmedikation eingesetzt wird. Grundgedanke dieser Therapie ist, dass die eingesetzten Präparate Verteilungsstörungen von Mineralien im Organismus regulieren, d.h. dafür sorgen, dass der richtige Mineralstoff zur richtigen Zeit in ausreichender Konzentration vorhanden ist. Einen klinisch feststellbaren Mineralstoffmangel können diese Präparate nicht ausgleichen!

Tab. 4 Schüßler-Salze mit Bezug zum Bewegungsapparat.

\begin{tabular}{|c|c|c|c|}
\hline Schüßler-Salz & Potenz & Gewebebezug & Einsatzgebiet \\
\hline $\begin{array}{l}\text { Calcium fluora- } \\
\text { tum Nr. } 1\end{array}$ & D12 & $\begin{array}{l}\text { Knochen, Sehnen, } \\
\text { Bänder, Gefäße }\end{array}$ & $\begin{array}{l}\text { Bei Jungtieren, die zur Über- } \\
\text { beanspruchung des Bewe- } \\
\text { gungsapparates neigen, nach } \\
\text { orthopädischen Operationen }\end{array}$ \\
\hline $\begin{array}{l}\text { Calcium } \\
\text { phosphoricum } \\
\text { Nr. } 2\end{array}$ & D6 & $\begin{array}{l}\text { Knochen, Zähne, } \\
\text { Zellkern }\end{array}$ & $\begin{array}{l}\text { Bei Wachstums- und Zahnungs- } \\
\text { beschwerden, Rachitis, nach } \\
\text { Frakturen }\end{array}$ \\
\hline $\begin{array}{l}\text { Natrium chlo- } \\
\text { ratum Nr. } 8\end{array}$ & D6 & $\begin{array}{l}\text { Körperflüssig- } \\
\text { keiten, Knorpel, } \\
\text { Knochen }\end{array}$ & $\begin{array}{l}\text { Knorpelschäden, Knacken in den } \\
\text { Gelenken }\end{array}$ \\
\hline Silicea Nr. 11 & D12 & $\begin{array}{l}\text { Bindegewebe, } \\
\text { Nerven, Haut, } \\
\text { Nägel }\end{array}$ & $\begin{array}{l}\text { Bindegewebsschwäche, } \\
\text { Bänderschwächen, Neuralgien }\end{array}$ \\
\hline $\begin{array}{l}\text { Calcium } \\
\text { carbonicum } \\
\text { Hahnemanni } \\
\text { Nr. } 22\end{array}$ & D12 & $\begin{array}{l}\text { Knochen, Schleim- } \\
\text { häute }\end{array}$ & $\begin{array}{l}\text { Wachsende Tiere mit Erschöp- } \\
\text { fungszuständen und Schleim- } \\
\text { hautentzündungen }\end{array}$ \\
\hline
\end{tabular}




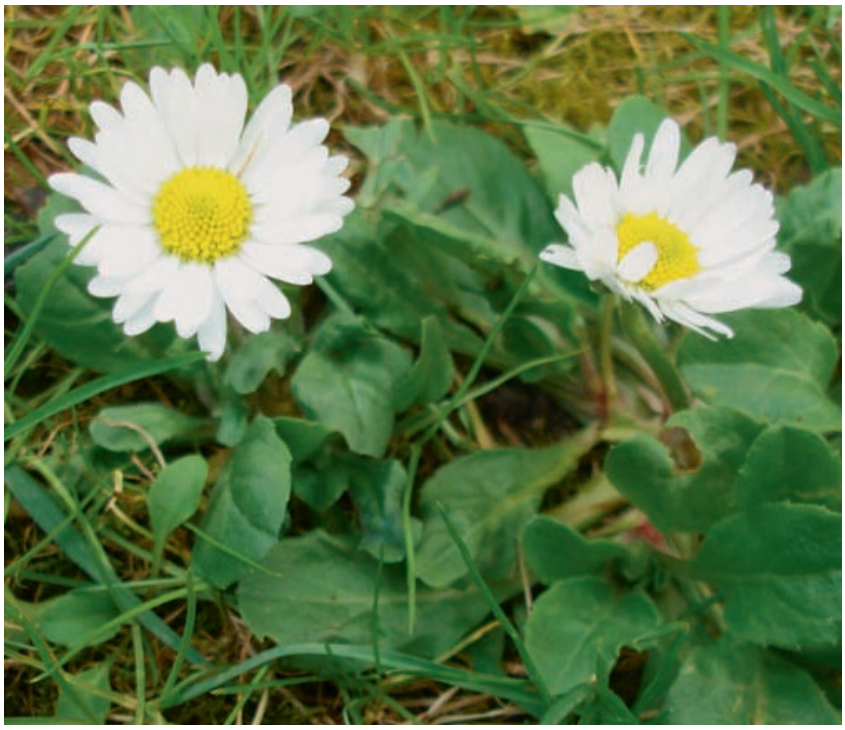

Abb. 5 Gänseblümchen-Bellis

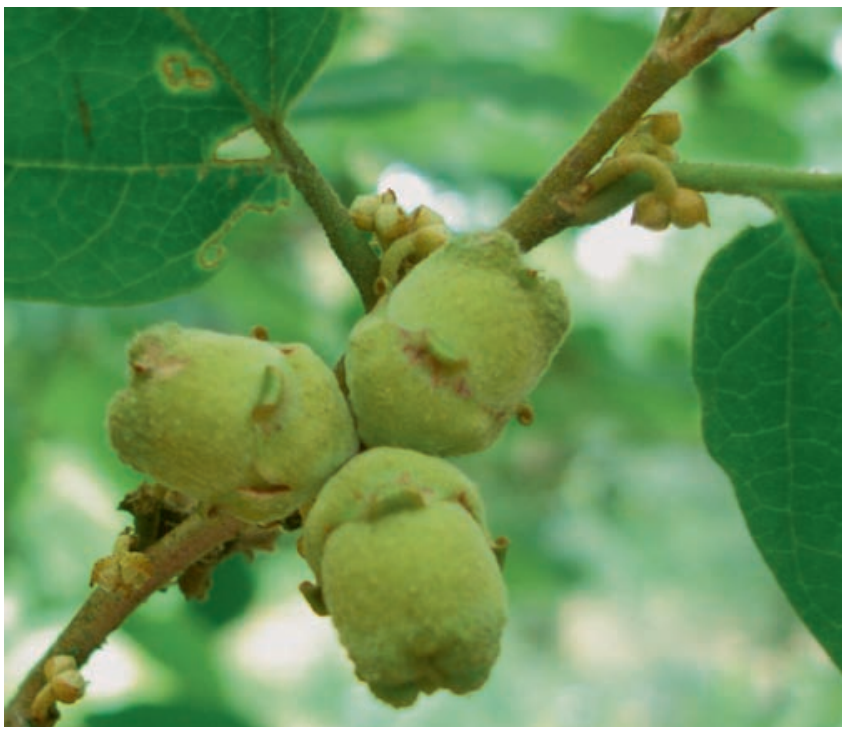

Abb. 6 Zaubernuss

\section{Phytotherapie}

Auch im Bereich der Pflanzenheilkunde gibt es Pflanzen, die analgetisch wirkende Substanzen enthalten und therapeutisch bei Schmerzen eingesetzt werden können. Vor allem dann, wenn andere potente
Schmerzmittel kontraindiziert sind oder Hunde mit Unverträglichkeit darauf reagieren, stellen sie eine gute Alternative dar.

So wirkt Teufelskralle (Harpagohytum procumens) bzw. die darin enthaltenen Iridoidglykoside antiphlogistisch und anal- getisch u.a. über eine Hemmung der 5Lipoxygenase. Alle Wirkmechanismen der Teufelskralle sind bisher pharmakologisch noch nicht aufgeklärt.

Das Gummiharz des indischen Weihrauches (Boswellia serrata) bzw. die darin enthaltenen Boswelliasäuren verfügt über 
eine ausgeprägte antiphlogistische Wirkung. In In-vitro-Studien konnte gezeigt werden, dass sowohl Lipoxygenasen als auch Cyclooxygenasen gehemmt werden.

Beinwell (Symphytum officinale) wirkt - äußerlich angewendet - abschwellend und antiphlogistisch durch Allantoin, Gerbstoffe, Rosmarinsäure, Triterpene und Sterole.

Leider gibt es in der Tiermedizin keine auf bestimmte Inhaltsstoffe standardisierten Phytopräparate zur Schmerztherapie. Die o.g. Heilpflanzen sind als Zusätze zu verschiedensten Nahrungsergänzungspräparaten für Hunde erhältlich, die in den von den Herstellern angegebenen Dosierungen verabreicht werden sollten.

\section{Low-Level-Lasertherapie}

Die Low-Level-Lasertherapie als Locusdolendi-Behandlung z. B. mit einem Infrarot-Laser ist sehr gut analgetisch wirksam bei neuralgischen und neuritischen Beschwerden und hat keinerlei Nebenwirkungen. Dabei summieren sich die analgetischen Laserwirkungen auf verschiedenen Ebenen: lokal entzündungshemmend, Ödeme vermindernd, ATP-Produktion und Wahrnehmungsschwelle für Schmerz erhöhend. Durch Blockade der Ionenpenetrationskanäle der Zellmembranen wird die Depolarisation von Nervenzellen erschwert. Durch die erhöhte ATP-Syntheseleistung wird der Nervenzelle Energie zur Verfügung gestellt um Na+-Ionen wieder ins extrazelluläre Mileu zu transpor- tieren. Beide Mechanismen wirken auf das Ruhemembranpotenzial der Nervenzelle stabilisierend, so dass Depolarisation und damit Auslösung eines Schmerzreizes erschwert sind.

Der schmerzlindernde Effekt des Infrarotlasers tritt bereits während der Behandlung ein und hält nach einer Erstbehandlung etwa 12-24 Stunden an. Nach Folgebehandlungen hält der analgetische Effekt dann auch länger an. Die meisten Gerätehersteller liefern mit ihren Geräten Tabellen mit, aus denen man die Dauer der Laserbestrahlung ablesen kann, die man nach Tab. 5 für bestimmte Wirkungen benötigt.

\section{Kombination Schul- und Regulationsmedizin}

Ist bei mittel- bis hochgradigen Lahmheiten nach Traumata ausgeschlossen, dass ein chirurgisches Vorgehen notwendig ist, kann die Erstbehandlung in der tierärztlichen Praxis mit bewährten kurz wirksamen nichtsteroidalen Antiphlogistika oder einem Infrarot-Laser erfolgen.

Der Tierhalter sollte unbedingt angewiesen werden, das Tier während der Gabe starker Schmerzmittel zu schonen bzw. in seiner Aktivität einzuschränken. Hat der akute hochgradige Schmerz sich nach 1-2 Tagen gebessert, kann eine Weiterbehandlung mit regulationsmedizinischen Präparaten erfolgen, z.B. mit einem auf den aktuellen Zustand passenden homöopathischen Einzelmittel oder mit einem Komplexmittel.

Tab. 5 Um mit dem Laser in der konventionellen Lasertherapie einen therapeutischen Effekt im Organismus zu erzielen, ist es notwendig, dem Gewebe Energie zuzuführen. Dafür gibt es gewisse Dosierungsrichtlinien:

\begin{tabular}{|c|c|c|c|}
\hline \multicolumn{2}{|l|}{ Gewünschter Effekt } & $\begin{array}{l}\text { Dosierung } \\
\text { Mensch }\end{array}$ & $\begin{array}{l}\text { Dosierung } \\
\text { Tier }\end{array}$ \\
\hline Analgesie & Muskel & $2-4$ joule $/ \mathrm{cm}^{2}$ & $2,5-5$ Joule $/ \mathrm{cm}^{2}$ \\
\hline & Gelenke & 4-8 Joule $/ \mathrm{cm}^{2}$ & 5-10 Joule/cm² \\
\hline Entzündungshemmender & Akut & $1-6$ joule $/ \mathrm{cm}^{2}$ & $1,25-7,5$ Joule $/ \mathrm{cm}^{2}$ \\
\hline Effekt & Chronisch & $4-8$ Joule $/ \mathrm{cm}^{2}$ & $5-10$ Joule $/ \mathrm{cm}^{2}$ \\
\hline Stoffwechselstimulation & & $3-6$ Joule $/ \mathrm{cm}^{2}$ & $3,75-7,5$ joule $/ \mathrm{cm}^{2}$ \\
\hline Vasomotorischer Effekt & & $1-3$ joule $/ \mathrm{cm}^{2}$ & $1,25-3,75$ Joule $/ \mathrm{cm}^{2}$ \\
\hline
\end{tabular}

Modifiziert nach: Ambronn/Muxeneder/Warnke: Laser- und Magnetfeldtherapie in der Tiermedizin

Diese Mittel werden so lange verabreicht, bis der Hund keine Lahmheit oder Abweichungen von seinem normalen Bewegungsmuster mehr zeigt, i.d.R. 7-14 Tage lang.

Online zu finden unter

http://dx.doi.org/10.1055/s-0030-1267783

\section{Literatur}

1 Ambronn, Muxeneder, Warnke: Laser- und Magnetfeldtherapie in der Tiermedizin Grundlagen und Anwendung; 2. Aufl. 1999; Sonntag Verlag

2 Homöopathisches Repetitorium, Ausgabe 2009, Fa. DHU (Deutsche HomöopathieUnion Karlsruhe)

3 Kübler H. Schüßler-Salze für Hunde. München: Gräfe und Unzer; 2007

4 Mezger J. Gesichtete homöopathische Arzneimittellehre. 12. Aufl. Stuttgart: Haug; 2005

5 Rakow B, Rakow M. Homöopathie in der Tiermedizin. Groß- und Kleintiere. Karlsbad: Aude sapere; 1997

6 Reichling J, Gachnian-Mirtscheva R, FraterSchröder M et al. Heilpflanzenkunde für Tierärzte. Heidelberg, Berlin: Springer; 2004

7 Reinhart E, Greef-Karstens C. Therapeutischer Index der Biologischen Tiermedizin. Baden-Baden: Aurelia Verlag; 2008

8 Reinhart E, Löw G, Greef-Karstens C. Kommentiertes Symptomenverzeichnis der Biologischen Tiermedizin. 3. Aufl. Baden-Baden: Aurelia-Verlag; 2006

9 Späth H, Löw G, Reinhart E. Gesunde Tiere durch Homöopathie und Antihomotoxische Medizin. 2. Aufl. Baden-Baden: Aurelia-Verlag; 2002

10 Weiss RV, Fintelmann V. Lehrbuch Phytotherapie. 12. Aufl. Stuttgart: Hippokrates; 2009

11 Westerhuis AH. Homöopathie für Hunde. München: Droemer Knaur; 2000

12 Wolff HG. Unsere Hunde - gesund durch Homöopathie. 14. Aufl. Stuttgart: Sonntag; 2002

Dr. med. vet. Heidi Kübler

Prakt. Tierärztin - Biologische Tiermedizin

Rudolf-Diesel-Straße 17

74182 Obersulm-Willsbach

E-Mail: Dr.Heidi.Kuebler@btm-nhv.de 